\title{
Intermediate and spin-liquid phase of the half-filled honeycomb Hubbard model
}

\author{
Qiaoni Chen, ${ }^{1}$ George H. Booth, ${ }^{1}$ Sandeep Sharma, ${ }^{1}$ Gerald Knizia, ${ }^{1,2}$ and Garnet Kin-Lic Chan ${ }^{1,3}$ \\ ${ }^{1}$ Department of Chemistry, Princeton University, Princeton, New Jersey 08544, USA \\ ${ }^{2}$ Institut fuer Theoretische Chemie, Universitaet Stuttgart, Pfaffenwaldring 55, D-70569 Stuttgart, Germany \\ ${ }^{3}$ Department of Physics, Princeton University, Princeton, New Jersey 08544, USA \\ (Received 23 February 2014; revised manuscript received 7 April 2014; published 28 April 2014)
}

\begin{abstract}
We obtain the phase diagram of the half-filled honeycomb Hubbard model with density matrix embedding theory, to address recent controversy at intermediate couplings. We use clusters from 2-12 sites and lattices at the thermodynamic limit. We identify a paramagnetic insulating state, with possible hexagonal cluster order, competitive with the antiferromagnetic phase at intermediate coupling. However, its stability is strongly cluster and lattice size dependent, explaining controversies in earlier work. Our results support the paramagnetic insulator as being a metastable, rather than a true, intermediate phase, in the thermodynamic limit.
\end{abstract}

DOI: 10.1103/PhysRevB.89.165134

PACS number(s): 75.10.Kt, 71.10.Fd, 71.30.+h

\section{INTRODUCTION}

Recently, there has been much debate over the zerotemperature phases of the Hubbard honeycomb model at half-filling. The accepted picture for many years was that for small interactions $U$, the system is semimetallic (SM) with a Dirac cone in the density of states [1]; at large $U$, the system is antiferromagnetically long-range ordered (AFM) [2,3]. However, Meng et al. proposed recently that an additional phase appears at intermediate couplings, arising from strong quantum fluctuations due to the low coordination number [4]. This phase has been further suggested to be a gapped spin liquid [5-8]. If present, this would be extremely significant, as spin liquids are not known to exist at half-filling without frustration [9-11], and would greatly advance the search for experimental realizations of 2D spin liquids, both in correlated honeycomb materials as well as optical lattices $[12,13]$.

A large number of numerical methods have been applied to study the honeycomb Hubbard model [1-4,11,14-32]. The work by Meng et al. used zero-temperature auxiliary-field (determinant) quantum Monte Carlo (AFQMC) [4]. These results were viewed with particular confidence because AFQMC has no sign problem in this model and thus correlations were treated "exactly," the only errors arising from using a finite size lattice of 648 sites. Subsequent to this, many reports of an intermediate phase have appeared using quantum cluster methods [33], such as cluster dynamical mean-field theory (CDMFT) with exact diagonalization (ED) (Liebsch [25] and He et al. [27]) and quantum Monte Carlo (CTQMC) solvers (Wu et al.) [29], and the variational cluster approximation (VCA) [28], including with several cluster shapes $[34,35]$. While these various quantum cluster methods differ in some details, they share a unifying Green function and self-energy functional formulation [36,37]; thus we refer to them collectively as Green function cluster (GFC) methods.

Despite these initial reports, there now appears increasing evidence that there may in fact be no spin-liquid phase $[11,21]$. The strongest hint is from Sorella et al. [21], who repeated Meng's AFQMC calculation with a larger lattice of 2592 sites. They found that at the increased lattice size, the region for an intermediate phase shrank significantly, suggesting weak (if any) evidence for a spin-liquid phase. Subsequently, a further
VCA calculation by Hassan and Senechal, in contrast to earlier quantum cluster calculations, also found no spin-liquid phase [11]. A recent VCA calculation [35] of Kane-Mele Hubbard model also suggests the nonmagnetic insulator observed previously [34] does not survive.

These conflicting reports raise important questions both for the physics of the honeycomb Hubbard model, and the numerical methods used to study it. Is there an intermediate phase, and is it a spin liquid? If not, what is observed in calculations which see an intermediate phase-is the system "close" to a spin liquid or some other state? Why do some quantum cluster calculations observe an intermediate phase and others not? And how do the various numerical approximations, such as finite lattice size in AFQMC, or finite cluster size in GFC methods, bias the calculations? These are the main questions we target in this work.

\section{DENSITY MATRIX EMBEDDING THEORY}

To answer these questions, we use a different quantum cluster method to those used previously - the recently proposed density matrix embedding theory (DMET) [38-41]. Note that DMET is not a variant of the GFC methods discussed above. In principle, GFC methods complement the exact AFQMC treatment because they work in the thermodynamic limit but only treat interactions exactly over a finite range determined by the impurity cluster size. In practice, however, solving the impurity problem within GFC methods involves further numerical approximations, such as bath discretization, or analytic continuation. Both require careful treatment to avoid affecting the physics [42-44]. Similar to GFC methods, DMET is a quantum cluster method that works (essentially) in the thermodynamic limit while treating interactions exactly over a finite range determined by the cluster size. In contrast, however, DMET has no bath discretization error by construction, and yields a quantum impurity problem where spectral functions can be practically computed without analytic continuation. This allows us to study the honeycomb Hubbard model at the thermodynamic limit with no further extraneous numerical approximations other than the finite range treatment of correlations. Then, by increasing the cluster size we can attempt convergence with respect to the range of correlations. 
DMET maps a large lattice problem to a quantum impurity plus bath problem, and is exact in the weak- and strongcoupling limits, as well as the limit of infinite cluster size. The main conceptual difference between DMET and GFC methods [45-48] is that the DMET bath is derived from the entanglement of the quantum state of the impurity cluster, rather than its single-particle Green function. DMET has several important technical advantages. If one is interested in thermodynamic properties, no Green functions need be computed; only the ground-state quantum impurity problem is solved, a significant computational savings. Further, since the DMET bath is defined from a Schmidt decomposition, the bath is the same size as the impurity with no bath discretization error, in contrast to the infinite bath in DMFT. Typically, an $n$-site cluster $(n>1)$ DMET calculation yields similar physics to an $n$-site CDMFT calculation that is converged with respect to the infinite bath parametrization [38-41]. However, the small $n$-site DMET Schmidt bath, together with the fact that the DMET embedding does not require Green's functions, means that ground-state DMET calculations are a fraction of the cost of comparable CDMFT calculations with diagonalization solvers. When computing DMET spectral functions, a further advantage (detailed below) is that the spectra are continuous without the need for artificial broadening, even with the finite bath. Finally, we note that although in this work we use exact diagonalization and density matrix renormalization group solvers, one could combine a determinantal QMC solver with DMET, which in sign-free models such as the half-filled honeycomb lattice would allow even larger clusters to be treated.

We now describe the DMET method briefly; for more details we refer to the original references [38-40]. We first choose an impurity cluster, cut from the underlying lattice. The underlying lattice is finite, but can trivially be made very large: in this study, we use lattices with more than 90000 sites. The "environment" lattice sites outside the cluster are then replaced by a bath. In ground-state DMET, the bath is defined with the help of a model lattice ground-state wave function $\left|\Psi^{(0)}\right\rangle$. In this study, $\left|\Psi^{(0)}\right\rangle$ is a (possibly spin-broken) Slater determinant (ground state) of a noninteracting lattice Hamiltonian $h+u$, where $h$ is the hopping matrix, and $u$ is a frequency-independent one-particle operator acting in each cluster cell on the lattice, analogous to a cluster self-energy. For ground-state DMET, the Schmidt decomposition of $\left|\Psi^{(0)}\right\rangle$ between the cluster and environment defines a bath space $\left\{\left|\beta^{0}\right\rangle\right\}$, of the same size as the impurity space. The quantum impurity Hamiltonian $H^{\prime}$ is then obtained by projecting a model lattice Hamiltonian $H_{\text {lat }}$ onto the quantum impurity plus bath space,

$$
H^{\prime}=P H_{\text {lat }} P=P\left(h+U_{\text {cluster }}+u\right) P,
$$

where $U_{\text {cluster }}$ indicates that, in $H_{\text {lat }}$, Hubbard interactions are present only on the cluster sites, as in CDMFT, while the one-particle operator $u$ is used for lattice sites outside the cluster, i.e., there are no interactions in the bath. Solving for the ground state of $H^{\prime}$ is a many-body problem with twice the degrees of freedom as the impurity cluster.

The resulting quantum impurity wave function yields expectation values, such as energies and correlation functions that approximate those of the original lattice problem. $u$ is adjusted to minimize the difference between the single-particle density matrix $\left\langle a_{i}^{\dagger} a_{j}\right\rangle$ of $H^{\prime}$ and the ground state of the model lattice Hamiltonian $h+u$ projected to the impurity plus bath space,

$$
\min _{u} \sum_{i j}\left|\left\langle a_{i}^{\dagger} a_{j}\right\rangle_{H^{\prime}}-\left\langle a_{i}^{\dagger} a_{j}\right\rangle_{h+u}\right|^{2}
$$

where $i, j$ range over the impurity plus bath sites, and the subscripts $H^{\prime}, h+u$ denote expectation values taken with respect to the interacting impurity plus bath ground state, and the lattice ground state, respectively. The minimization ensures that the noninteracting lattice wave function and the associated embedding best updates to reflect information about the impurity correlations, as measured by the one-particle density matrices. The self-consistency condition is also analogous to the self-consistent update of the self-energy in DMFT, which requires the local Green's functions of the lattice and the impurity to match.

To obtain spectra in DMET a modified procedure is used, where the ground-state bath space $\left\{\left|\beta^{0}\right\rangle\right\}$ is augmented to reproduce dynamical properties. For example, to compute the local single-particle Green function, we consider the response vector of the model lattice wave function,

$$
\left|\Psi^{(1)}(\omega)\right\rangle=\frac{1}{\omega+\mu-h+u+i 0} a_{\alpha}^{(\dagger)}\left|\Psi^{(0)}\right\rangle
$$

and obtain a set of additional bath states from the Schmidt decomposition of $\left|\Psi^{(1)}(\omega)\right\rangle$, leading to a total bath space $\left\{\left|\beta^{0}\right\rangle \oplus\left|\beta^{1}(\omega)\right\rangle\right\}$. The cluster Green function is determined by solving, at each frequency, the response problem for $H_{\text {lat }}$ projected into the impurity plus dynamical bath space. Although the bath space is finite, it changes with frequency; thus the finite impurity model produces a continuous spectrum along the real axis without artificial broadening. Note that, in this work, we do not take any special steps to restore translational symmetry when computing the spectral functions.

\section{HONEYCOMB HUBBARD MODEL}

We now turn to applying DMET to the honeycomb Hubbard model. To identify different phases we monitor several quantities. The SM phase is characterized by a vanishing single-particle gap and a Dirac cone at low energies in the single-particle density of states $A(\omega)$. The AFM phase is characterized by nonvanishing staggered magnetization, $M=\frac{1}{N} \sum_{i j}(-1)^{i+j}\left(n_{i \uparrow}-n_{j \downarrow}\right)$, a nonzero single-particle gap (which grows with increasing $U$ ), and a vanishing spin gap. The proposed intermediate gapped phase is identified by a nonvanishing single particle and spin gap without long-range AFM order. We further check for spin-liquid character from the correlation functions. Meng et al. [4] further suggested that the intermediate phase can be identified from the gradient of $d T / d U$, where $T$ is the kinetic energy. Not all the above quantities have been of equal focus in earlier studies; AFQMC studies report energies and gaps but not spectral functions, while GFC calculations report spectral quantities but not energies. Here, using DMET, we study energies as well as gaps and spectral functions.

To study the effect of cluster size and shape, we perform calculations with $N_{c}=2-12$ cluster sites; these are shown 


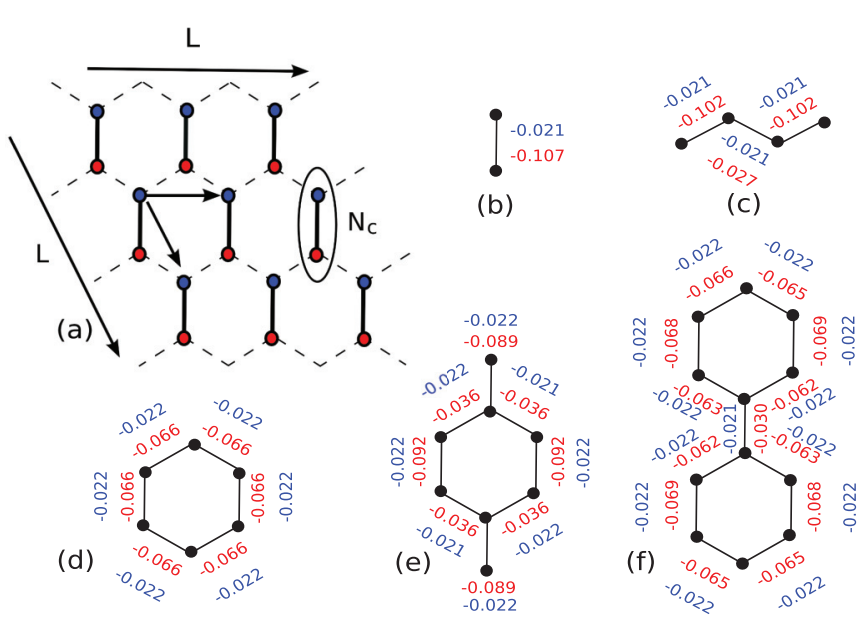

FIG. 1. (Color online) (a) DMET embeds clusters of $N_{c}$ sites in the underlying honeycomb lattice of $2 L^{2}$ sites. (b)-(f) Cluster shapes in our study, for $N_{c}=2-12$. Also shown is the nearest-neighbor spin-spin correlation, $\left\langle S_{i}^{z} S_{j}^{z}\right\rangle$. Blue numbers are from $U=1.5 t$ in the semimetal $(\mathrm{SM})$ phase; red numbers are from $U=8.4 t$ in the paramagnetic insulator (PMI) phase.

in Fig. 1. For $N_{c}=2-6$ (4-12 sites with the bath) we use an exact diagonalization solver; for $N_{c}=8-12$ (16-24 sites with bath) we use a density matrix renormalization group (DMRG) solver [49], keeping up to 2000 states, sufficient for quasiexactness. It is important to distinguish cluster size $N_{c}$ from the size of the lattice $\left(2 L^{2}\right)$ in which the cluster is embedded. To study finite-size scaling effects of the underlying lattice, we use embedding lattices with $L \times L$ supercells $\left(2 L^{2}\right.$ sites), with $L=12-216$ (up to over 90000 sites). The smaller $L$ calculations allow direct comparison to finite AFQMC calculations: for comparison, Meng et al. used up to $L=18$; Sorella et al. used up to $L=36$.

We start by scanning the phase diagram as a function of $U$, allowing spontaneous antiferromagnetism to develop. The single-particle gap, magnetization, and density of states for $N_{c}=2-12$ are shown in Fig. 2 . We see at small $U$ the single-particle gap vanishes and the density of states displays a low-energy Dirac cone, clearly demonstrating that the system is in the SM phase. As we increase $U$ beyond a critical $U_{\mathrm{AFM} 1}$ an AFM solution to the DMET equations appears and a gap opens in the spectral function (Fig. 2). Note that $U_{\mathrm{AFM} 1}$ is the earliest point at which the AFM solution can be found but this does not indicate a thermodynamic phase transition at this point, which requires a more detailed examination of the energies, discussed below. At $U_{\mathrm{AFM} 1}$, the single-particle gap and magnetization vanish simultaneously. Fitting $M=$ $\left|U-U_{\mathrm{AFM} 1}\right|^{\beta}$ to the $N_{c}=6$ data gives a critical exponent $\beta=0.72$, compared with $0.80 \pm 0.04$ from the AFQMC calculations of Sorella et al., and $\beta=1$ from mean field. $U_{\mathrm{AFM} 1}$ shows significant cluster and lattice size dependence, as seen in Fig. 2 ; for $N_{c}=6, L=12-216, U_{\mathrm{AFM} 1}$ decreases from 3.329 to 3.198 , while at $L=216$, for $N_{c}=2-12$, $U_{\mathrm{AFM} 1}$ increases from 2.92 to 3.2 . Our $U_{\mathrm{AFM} 1}=3.2$ for $N_{c}=12, L=216$ is somewhat lower than the recent AFQMC result $U_{\mathrm{AFM} 1}=3.869$ of Sorella et al. [21]. The lattice size dependence of $U_{\mathrm{AFM} 1}$ gives an estimate of the finite size error in
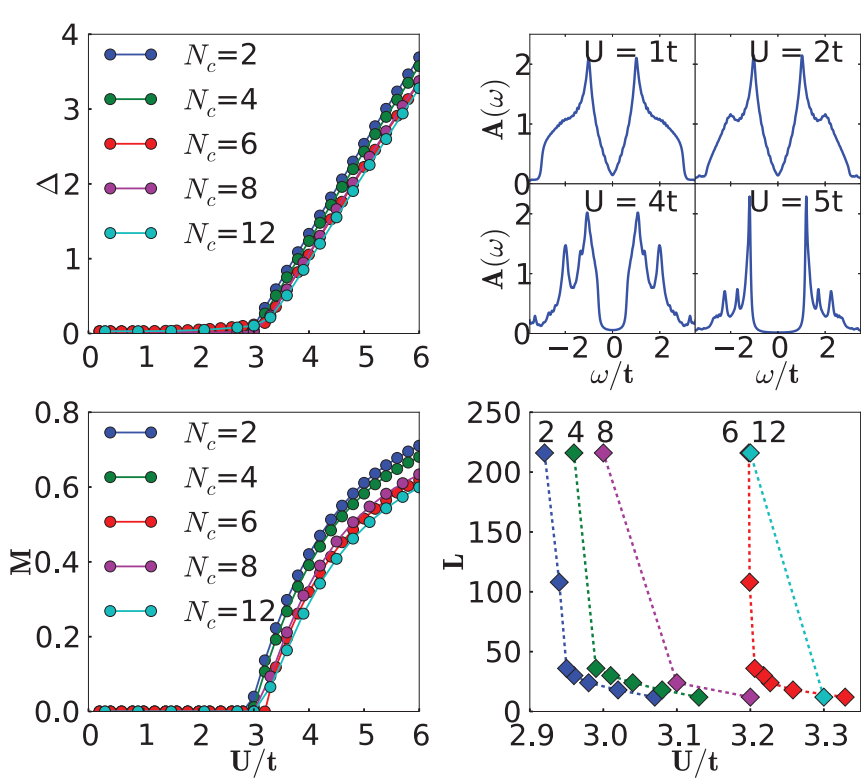

FIG. 2. (Color online) DMET calculations allowing spontaneous AFM order. Top left: single-particle gap against $U$ for cluster sizes $N_{c}=2-12$. As $U$ increases, a gap spontaneously opens at $U_{\mathrm{AFM}}$, just after $U=3$. Top right: local density of states when $N_{c}=6$, calculated from spectral DMET [40], showing the Dirac cone in the SM phase. Bottom left: staggered magnetization against $U$ for $N_{c}=2-12$. The staggered magnetization appears at the same point as the opening of the gap. Bottom right: $U_{\mathrm{AFM} 1}$ as a function of lattice size $L=12-216$ (288-93312 sites) and cluster size $\left(N_{c}=2-12\right)$.

Sorella et al.'s $L=36$ calculations; from $L=36-216, U_{\mathrm{AFM} 1}$ decreases by $\sim 0.01$.

A quantitative check of the accuracy of our calculations is to directly compare the energies for a given finite size lattice with the exact AFQMC energies for the same lattice size. (While we do not argue that energies provide the complete picture, comparing to the exact AFQMC results on finite lattices is a concrete benchmark.) This comparison for total energies is shown in Fig. 3. We have compared over only a limited range of $U$ as this is where we had access to the QMC data [21]. However, theoretically and numerically it is known that the error in the DMET energies vanishes exactly at weak and strong coupling; the largest errors are near phase transitions [38], the region tested in Fig. 3. Even in this challenging region, the cluster DMET total energies appear extremely good, as expected from earlier benchmarks [38,39,41]; with $N_{c}=12$ the energies are within $0.2 \%$ of the exact results. The more sensitive kinetic-energy derivative $d T / d U$ reported by Meng et al. (and obtained from Sorella et al.) is also shown in Fig. 4. Here we find that although Meng et al. argued that the two changes in curvature in $d T / d U$ near $U=3.5,4.3$ indicate an intermediate phase, we also observe two changes in curvature in our cluster DMET curve, at similar places, with just a SM and AFM phase, showing this is not a good diagnostic for an intermediate phase.

We now consider the intermediate $U$ region and look for evidence of possible metastable intermediate phases. To do so, we restrict our DMET calculations to paramagnetic phases and increase $U$. Interestingly, as $U$ is increased, we observe two 

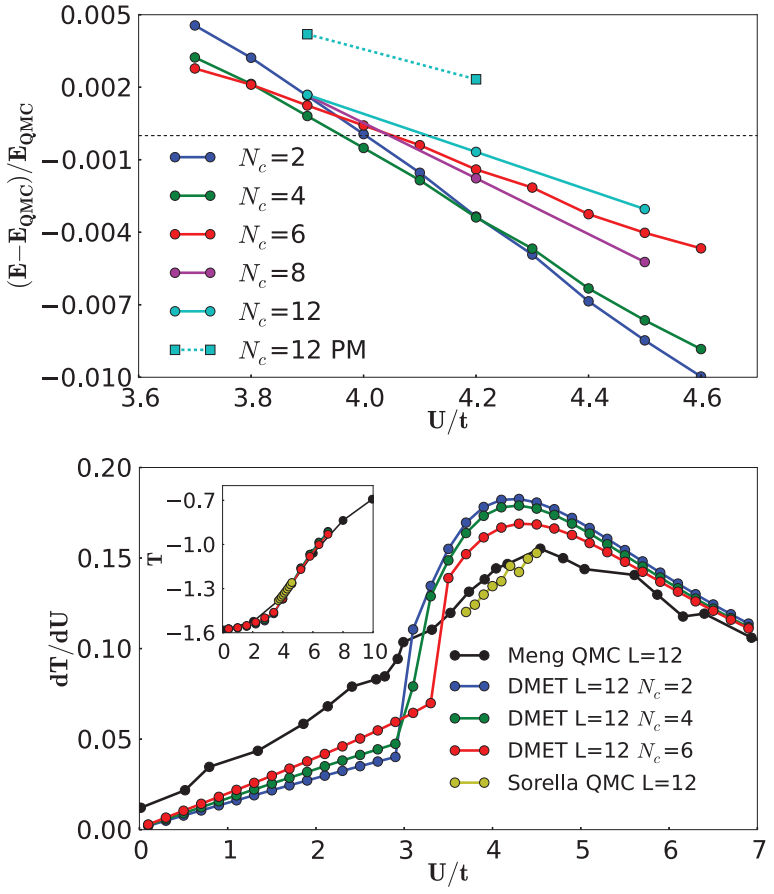

FIG. 3. (Color online) Top: DMET energy and Sorella et al.'s finite lattice AFQMC energy at intermediate $U$ for $L=12$ (288 sites) as a function of cluster size $N_{c}=2-12$. This is the most challenging regime for DMET, but the energy comparison is very favorable. PM denotes paramagnetic solution. Bottom: derivative of kinetic energy as a function of $U / t$. Overlaid are results of Meng et al. and Sorella et al. for $L=12$ [4]. Inset: kinetic energy density as a function of $U$, showing good agreement between the DMET and numerically exact AFQMC [4]. DMET qualitatively reproduces two changes in curvature (near $U=3.5$ and $U=4.3$ ) cited as evidence of an intermediate phase by Meng et al., although no intermediate phase is observed.

kinds of paramagnetic transitions depending on cluster shape: for $N_{c}=2,4,8$, there is a first-order transition to a gapped phase, while for $N_{c}=6,12$, there is a second-order transition: the gap opens continuously. The qualitative difference in the transition between clusters of different shapes indicates we are still somewhat far from convergence with respect to cluster size, however, in either case, we find a paramagnetic insulating phase (PMI) at large $U$. The finding of a PMI phase is not unexpected; preceding GFC calculations have identified a similar phase, typically identified as a Mott insulator. We can compare the $N_{c}=6$ DMET PMI gaps directly to earlier sixsite paramagnetic GFC calculations (see [1] for a summary). The agreement at larger $U$ is especially good. Note, further, that we find the paramagnetic transition $U_{\mathrm{PM}}$ is very sensitive to cluster size, as seen in Fig. 5. In fact, the qualitatively different transition for $N_{c}=6,12$ and $N_{c}=2,4,8$ suggests that the cluster treatment is converging on two different kinds of PMI phase, with different order. We return to this point further below.

Hassan and Senechal conjectured that the purported spinliquid phase in the honeycomb Hubbard model $i s$ in fact the PMI phase in a quantum cluster calculation [11]. We now examine this conjecture. To identify whether the PMI phase
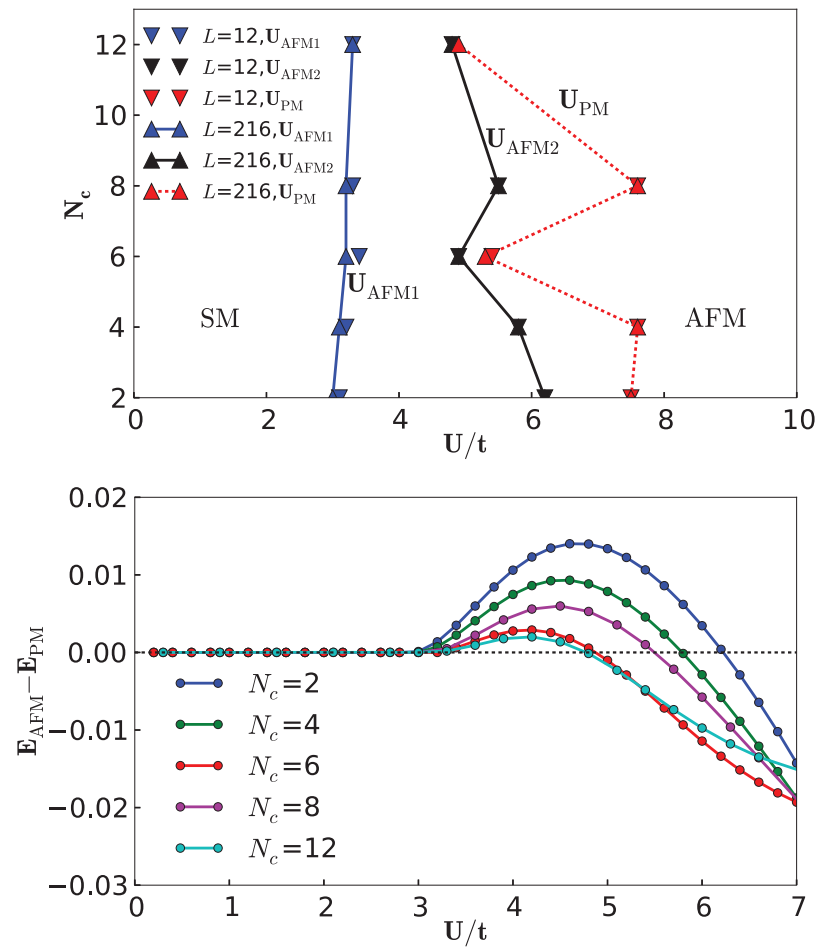

FIG. 4. (Color online) Top: phase transition points for cluster sizes $2-12$, and two lattice sizes $L=12,216$. $U_{\mathrm{AFM} 1}$ corresponds to opening of the gap. $U_{\mathrm{AFM} 2}$ corresponds to the thermodynamic transition to the $\mathrm{AFM}$ phase. $\left[U_{\mathrm{AFM} 1}, U_{\mathrm{AFM} 2}\right]$ is a coexistence region for the AFM phase and SM phase, although this region appears to vanish with increasing cluster size. $U_{\mathrm{PM}}$ is the transition to the PMI if AFM order is not allowed to develop. The transition is first order for $N_{c}=2,4,8$ and second order for $N_{c}=6,12$. Note that for $N_{c}=6,12$, $U_{\mathrm{PM}}$ is very close to $U_{\mathrm{AFM} 2}$ indicating that the $\mathrm{PMI}$ is very competitive with the AFM phase for these cluster shapes. Bottom: ground state energy difference of PM and AFM solutions. The positive region is the coexistence region.

is a true intermediate phase, we must check its stability in the presence of antiferromagnetism. In the square lattice, antiferromagnetism order appears at infinitesimal $U$ before the PMI phase is reached. The honeycomb Hubbard model differs in this regard because AFM order develops at finite $U_{\mathrm{AFM} 1}$, which could in principle be larger than $U_{\mathrm{PM}}$. In Fig. 4 we show the energy of the AFM phase relative to the SM/PMI phases as a function of cluster and lattice size. Here, we find that for our cluster sizes the transition between SM and AFM does not in fact occur at $U_{\mathrm{AFM} 1}$; rather there is a first-order coexistence region between $U_{\mathrm{AFM} 1}, U_{\mathrm{AFM} 2}$, where $U_{\mathrm{AFM} 2}$ is strongly cluster size (and to a lesser degree lattice size) dependent (Fig. 4). As the cluster size increases, the first-order coexistence region decreases, and it appears that in the limit of infinite cluster size the true AFM transition is second order (Fig. 4). Further, in all instances we have studied, $U_{\mathrm{AFM} 2}<U_{\mathrm{PM}}$ at the same cluster size. This means that there is in fact no stable intermediate paramagnetic phase. However, for the special cluster sizes $N_{c}=6,12$, the PMI phase appears particularly competitive in the coexistence region, as seen from Fig. 4. Although a sharp PM phase transition cannot be observed for $N_{c}=6,12$ (since there is a small gap even at small $U$ due to breaking 


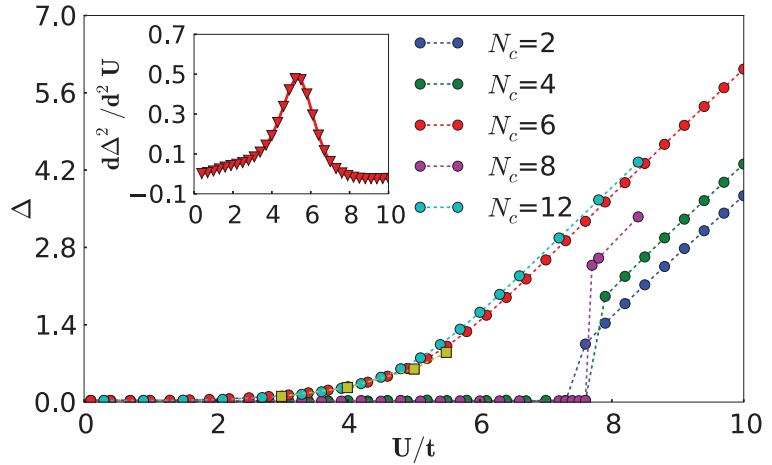

FIG. 5. (Color online) Single particle gap of paramagnetic solution with cluster sizes for $L=216$. For $N_{c}=2,4,8$ the transition is first order; for $N_{c}=6,12$ the transition is continuous. Inset: second derivative of the gap for $N_{c}=6,12$; we identify $U_{\mathrm{PM}}$ from the peak. Yellow squares: six-site CDMFT calculations from Ref. [1].

of translational symmetry in a cluster approach as discussed in [1]) there is a very clear change in curvature of the gap $d^{2} \Delta / d U^{2}$. This defines an effective $U_{\mathrm{PM}}$ that would become a sharp transition for larger clusters made of the same hexagonal cells as $N_{c}=6$. For these clusters, $U_{\mathrm{AFM} 2}$ is then very close to $U_{\mathrm{PM}}$, however, the uncertainty in $U_{\mathrm{PM}}$ due to the continuous opening of the gap means that a small change in definition of $U_{\mathrm{PM}}$ could yield $U_{\mathrm{PM}}<U_{\mathrm{AFM} 2}$, and a conclusion that there is a true paramagnetic intermediate phase. In addition, the very small energy difference between the AFM and PMI phase in the coexistence region and the closeness of $U_{\mathrm{AFM} 2}$ and $U_{\mathrm{PM}}$ provides a basis to explain the conflicting observations, in both AFQMC and in GFC calculations, of an intermediate phase near the antiferromagnetic transition: small changes in calculation parameters can selectively stabilize the PMI phase. Our arguments therefore support the interpretation of Hassan et al. that observations of the "intermediate" phase can be identified with the PMI state in quantum cluster calculations.

What is the nature of the paramagnetic state for $N_{c}=6,12$ ? Although this "intermediate phase" is gapped without longrange magnetic order, this does not mean that it is a spin liquid; another obvious candidate would be some kind of valencebond crystal. The particular stabilization of the paramagnetic insulator for the hexagonal based clusters $N_{c}=6,12$, suggests that it is associated with a hexagonal cluster (Kekule valencebond crystal) order. The annotations in Fig. 1 show the spinspin correlation functions $\left\langle S_{i}^{z} S_{j}^{z}\right\rangle$. Although these correlation functions in cluster DMET (as in CDMFT) are not guaranteed to preserve translational invariance, the pattern of translational invariance breaking can be revealing of an underlying order. Indeed, the spin-correlation functions for $N_{c}=12$ confirm that a hexagonal cluster order develops in this PMI phase (note there is no symmetry breaking in the corresponding SM phase for $N_{c}=12$, and further that the single $N_{c}=6$ cluster shows no evidence of dimerization). A similar hexagonal cluster order has been established in the large $N$ limit of the SU(N) exchange model [50] and $J_{1}-J_{2}$ model [51]. Intriguingly the hexagonal order has also been implicated as a real instability of graphene under strain [52]. The (uncompetitive) PMI phase for $N_{c}=$ 2,4,8 develops a simpler dimer order. Although this dimer ordered phase is stabilized by the special shape of the clusters for $N_{c}=2,4,8$, it is never competitive with the AFM phase.

\section{CONCLUSIONS}

In conclusion, we have carried out cluster DMET calculations, as a function of cluster and lattice size, to elucidate the phase diagram of the half-filled Hubbard honeycomb model. Our detailed calculations find that, at intermediate couplings, there is a metastable paramagnetic insulating phase, that is very competitive with the antiferromagnetic phase. This insulating phase displays an associated hexagonal cluster order. The closeness of the two phases at intermediate couplings means that small changes in calculational details can significantly affect their relative stability, and this explains the large number of conflicting results regarding the intermediate phase. It further seems likely that the intermediate paramagnetic phase can be stabilized by introducing modest frustration. Finally, our work demonstrates the potential of the DMET methodology, which allows computation of both energies and spectral functions at the thermodynamic limit, without incurring additional numerical artifacts.

\section{ACKNOWLEDGMENTS}

We gratefully acknowledge S. Sorella and Y. Otsuka for providing the energy data from their QMC calculations. The main funding for this work was from the U.S Department of Energy, DE-SC0008624 and DE-SC0010530. Additional funding was provided through the DOE-CMCSN program DESC0006613 through support of G.K. Initial funding for Q.C. was provided by a seed grant from the NSF, MRSEC Award No. NSF-DMR 0819860.
[1] A. Liebsch and W. Wu, Phys. Rev. B 87, 205127 (2013).

[2] S. Sorella and E. Tosatti, Europhys. Lett. 19, 699 (1992).

[3] L. Martelo, M. Dzierzawa, L. Siffert, and D. Baeriswyl, Z. Phys. B 103, 335 (1997).

[4] Z. Y. Meng, T. C. Lang, S. Wessel, F. F. Assaad, and A. Muramatsu, Nature (London) 464, 847 (2010).

[5] F. Wang, Phys. Rev. B 82, 024419 (2010).

[6] C. Xu, Phys. Rev. B 83, 024408 (2011).

[7] Y.-M. Lu and Y. Ran, Phys. Rev. B 84, 024420 (2011).

[8] B. K. Clark, arXiv:1305.0278.
[9] L. Balents, Nature (London) 464, 199 (2010).

[10] I. Kimchi, S. A. Parameswaran, A. M. Turner, F. Wang, and A. Vishwanath, Proc. Natl. Acad. Sci. USA 110, 16378 (2013).

[11] S. R. Hassan and D. Sénéchal, Phys. Rev. Lett. 110, 096402 (2013).

[12] K. S. Novoselov, A. K. Geim, S. V. Morozov, D. Jiang, Y. Zhang, S. V. Dubonos, I. V. Grigorieva, and A. A. Firsov, Science 306, 666 (2004).

[13] R. Walters, G. Cotugno, T. H. Johnson, S. R. Clark, and D. Jaksch, Phys. Rev. A 87, 043613 (2013). 
[14] J. E. Drut and T. A. Lähde, Phys. Rev. Lett. 102, 026802 (2009).

[15] J. E. Drut and T. A. Lähde, Phys. Rev. B 79, 165425 (2009).

[16] T. Paiva, R. T. Scalettar, W. Zheng, R. R. P. Singh, and J. Oitmaa, Phys. Rev. B 72, 085123 (2005).

[17] S.-Q. Su, K.-M. Tam, and H.-Q. Lin, Phys. Rev. B 80, 104517 (2009).

[18] K. L. Lee, K. Bouadim, G. G. Batrouni, F. Hébert, R. T. Scalettar, C. Miniatura, and B. Grémaud, Phys. Rev. B 80, 245118 (2009).

[19] B. K. Clark, D. A. Abanin, and S. L. Sondhi, Phys. Rev. Lett. 107, 087204 (2011).

[20] H.-Y. Yang, A. Albuquerque, S. Capponi, A. M. Luchli, and K. P. Schmidt, New J. Phys. 14, 115027 (2012).

[21] S. Sorella, Y. Otsuka, and S. Yunoki, Sci. Rep. 2, 992 (2012).

[22] M.-T. Tran and K. Kuroki, Phys. Rev. B 79, 125125 (2009).

[23] S. A. Jafari, Eur. Phys. J. B 68, 537 (2009).

[24] W. Wu, Y.-H. Chen, H.-S. Tao, N.-H. Tong, and W.-M. Liu, Phys. Rev. B 82, 245102 (2010).

[25] A. Liebsch, Phys. Rev. B 83, 035113 (2011).

[26] K. Seki and Y. Ohta, J. Korean Phys. Soc. 62, 2150 (2013).

[27] R.-Q. He and Z.-Y. Lu, Phys. Rev. B 86, 045105 (2012).

[28] S.-L. Yu, X. C. Xie, and J.-X. Li, Phys. Rev. Lett. 107, 010401 (2011).

[29] W. Wu, S. Rachel, W.-M. Liu, and K. Le Hur, Phys. Rev. B 85, 205102 (2012).

[30] T. Li, Europhys. Lett. 93, 37007 (2011).

[31] A. Giuliani and V. Mastropietro, Commun. Math. Phys. 293, 301 (2010)

[32] F. F. Assaad and I. F. Herbut, Phys. Rev. X 3, 031010 (2013).

[33] T. Maier, M. Jarrell, T. Pruschke, and M. H. Hettler, Rev. Mod. Phys. 77, 1027 (2005).

[34] J. C. Budich, R. Thomale, G. Li, M. Laubach, and S.-C. Zhang, Phys. Rev. B 86, 201407 (2012).
[35] M. Laubach, J. Reuther, R. Thomale, and S. Rachel, arXiv:1312.2934.

[36] M. Potthoff, Eur. Phys. J. B 32, 429 (2003).

[37] M. Potthoff, Eur. Phys. J. B 36, 335 (2003).

[38] G. Knizia and G. K.-L. Chan, Phys. Rev. Lett. 109, 186404 (2012).

[39] G. Knizia and G. K.-L. Chan, J. Chem. Theory Comput. 9, 1428 (2013).

[40] G. H. Booth and G. Kin-Lic Chan, arXiv:1309.2320.

[41] I. W. Bulik, G. E. Scuseria, and J. Dukelsky, Phys. Rev. B 89, 035140 (2014).

[42] D. Zgid, E. Gull, and G. K.-L. Chan, Phys. Rev. B 86, 165128 (2012).

[43] N. Lin, C. A. Marianetti, A. J. Millis, and D. R. Reichman, Phys. Rev. Lett. 106, 096402 (2011).

[44] E. Gull, A. J. Millis, A. I. Lichtenstein, A. N. Rubtsov, M. Troyer, and P. Werner, Rev. Mod. Phys. 83, 349 (2011).

[45] W. Metzner and D. Vollhardt, Phys. Rev. Lett. 62, 324 (1989).

[46] A. Georges and W. Krauth, Phys. Rev. Lett. 69, 1240 (1992).

[47] G. Kotliar, S. Y. Savrasov, K. Haule, V. S. Oudovenko, O. Parcollet, and C. A. Marianetti, Rev. Mod. Phys. 78, 865 (2006).

[48] A. Georges, G. Kotliar, W. Krauth, and M. J. Rozenberg, Rev. Mod. Phys. 68, 13 (1996).

[49] S. Sharma and G. K.-L. Chan, J. Chem. Phys. 136, 124121 (2012).

[50] T. C. Lang, Z. Y. Meng, A. Muramatsu, S. Wessel, and F. F. Assaad, Phys. Rev. Lett. 111, 066401 (2013).

[51] R. F. Bishop, P. H. Y. Li, D. J. J. Farnell, and C. E. Campbell, J. Phys.: Condens. Matter 24, 236002 (2012).

[52] C. A. Marianetti and H. G. Yevick, Phys. Rev. Lett. 105, 245502 (2010). 